\title{
A non-stationary subdivision scheme for curve interpolation
}
M. K. Jena*
P. Shunmugaraj ${ }^{\dagger}$
P. C. $\operatorname{Das}^{\ddagger}$

(Received 12 June 2001; revised 11 October 2002)

\begin{abstract}
We present a non-stationary subdivision scheme for interpolating a set of given data points. The scheme is a generalisation of the four point subdivision scheme of Dyn and Levin to the non-stationary case. This scheme reproduces elements of the linear space spanned by $1, \cos (\alpha x)$ and $\sin (\alpha x)$. Moreover if the initial data lies on a $C^{2}(\mathbb{R})$ function, then the limit function of the scheme approximates the original function quadratically.
\end{abstract}

\section{Contents}

\section{Introduction}

*Department of Mathematics, Indian Institute of Technology, Kanpur-208016, InDiA.

†as above. mailto:psraj@iitk.ac.in

$\ddagger$ as above. mailto:pcdas@iitk.ac.in

${ }^{0}$ See http: //anziamj . austms .org. au/V44/E031 for this article, (c) Austral. Mathematical Soc. 2003. Published February 14, 2003 ISSN 1446-8735 
2 Preliminaries and definitions

E218

3 The subdivision scheme

E220

4 Basic limit function

E224

5 Reconstruction of functions

E228

6 Order of approximation

E231

7 Discussion

E233

References

E234

\section{Introduction}

Subdivision schemes are important and efficient tools for generating curves and surfaces iteratively from a finite set of points. Initially subdivision procedures were used to construct uniform and non-uniform B-spline curves. Now they have become a subject of study in their own right with a variety of applications. For example [8], through the theory of multiresolution analysis, subdivision can be used to create functions such as wavelets that have no analytic forms. Recently subdivision schemes have been applied to solve problems in fluid flow [9].

Each subdivision scheme is associated with a mask [2,3] and is called stationary if the same mask is used in each step of the iteration. However, interest has been growing in non-stationary schemes.

The objective of the present work is to develop a non-stationary binary subdivision scheme whose limit curve interpolates a given 
set of data points. The scheme is a generalisation of a well-known interpolatory subdivision scheme by Dyn and Levin [4] (also by Dubuc [1]). Our scheme reproduces the elements of the linear space spanned by $1, \cos (\alpha x)$ and $\sin (\alpha x)$. This scheme can also be used to approximate smooth functions effectively.

The paper is organised as follows. In Section 2 some definitions and known results about non-stationary subdivision schemes are presented. In Section 3 our new subdivision scheme is introduced and its convergence is studied. Some geometric properties of the basic limit functions are presented in Section 4. In Section 5 we show that the scheme reconstructs a certain class of trigonometric polynomials. Finally, the quadratic order of approximation of the limit function is shown in Section 6.

\section{Preliminaries and definitions}

We present some previously established definitions and results on subdivision schemes which are used in what follows.

Given a set of control points $P^{0}=\left\{p_{i} \in \mathbb{R}^{d} \mid i \in \mathbb{Z}\right\}$ at level 0 , a subdivision scheme $\left\{S_{a_{k}}\right\}_{k \geq 1}$ generates a new set of control points $P^{k}=\left\{p_{i}^{k} \mid i \in \mathbb{Z}\right\}$ at the $k$ th level by a subdivision rule:

$$
p_{i}^{k}=\left(S_{a_{k}} P^{k-1}\right)_{i}=\left(S_{a_{k}} S_{a_{k-1}} \cdots S_{a_{1}} P^{0}\right)_{i}=\sum_{j=1}^{\infty} a_{i-2 j}^{(k)} p_{j}^{k-1}, \quad i \in \mathbb{Z}
$$

where the set $\left\{a_{i}^{(k)} \mid i \in \mathbb{Z}, a_{i}^{(k)} \neq 0\right\}$ is finite for every $k \in \mathbb{N}$. It is sufficient to study the subdivision scheme for the initial points $p_{i}^{0} \in$ $\mathbb{R}$ as the subdivision scheme is applied componentwise.

The set $\mathbf{a}^{(\mathbf{k})}:=\left\{a_{i}^{(k)} \mid i \in \mathbb{Z}\right\}$ of coefficients is called the mask at the $k$ th level of the subdivision scheme. If the mask is independent 
of $k$, then the scheme is called stationary, otherwise it is called non-stationary. To each subdivision scheme $\left\{S_{a_{k}}\right\}$ defined by the mask $\left\{a_{i}^{k}\right\}_{k \geq 1}$, we assign the polynomial

$$
a_{k}(z)=\sum_{i \in \mathbb{Z}} a_{i}^{(k)} z^{i},, \quad k \geq 1
$$

called the $k$ th level characteristic polynomial of the algorithm.

For $k=0,1, \ldots$ the points $p_{i}^{k}, i \in \mathbb{Z}$ are assigned to the mesh points $2^{-k} j, j \in \mathbb{Z}$ respectively.

Definition 1 [3] A subdivision scheme $\left\{S_{a_{k}}\right\}$ is said to be $C^{m}$ if for every initial data $P^{0} \in l^{\infty}$ there exists a limit function $f \in C^{m}(\mathbb{R})$ such that

$$
\lim _{k \rightarrow \infty} \sup _{\alpha \in \mathbb{Z}}\left|P_{\alpha}^{k}-f\left(2^{-k} \alpha\right)\right|=0
$$

and $f \neq 0$ for some initial data $P^{0}$.

Definition 2 [3] Let $\mathbf{a}^{(\mathbf{k})}$ be the mask at the kth level of the subdivision scheme $\left\{S_{a_{k}}\right\}$. Then the set $\left\{i \in \mathbb{Z} \mid a_{i}^{(k)} \neq 0\right\}$ is called the support of the mask $\mathbf{a}^{(\mathbf{k})}$.

Definition 3 [3] Two subdivision schemes $\left\{S_{a_{k}}\right\}$ and $\left\{S_{b_{k}}\right\}$ are asymptotically equivalent if

$$
\sum_{k=1}^{\infty}\left\|S_{a_{k}}-S_{b_{k}}\right\|_{\infty}<\infty
$$

where $\left\|S_{a_{k}}\right\|_{\infty}=\max \left\{\sum_{\alpha \in \mathbb{Z}}\left|a_{2 \alpha}^{(k)}\right|, \sum_{\alpha \in \mathbb{Z}}\left|a_{2 \alpha+1}^{(k)}\right|\right\}$.

The following result [3] relates the convergence of a non-stationary scheme to its asymptotically equivalent stationary scheme. 
Theorem 4 Let $\left\{S_{a_{k}}\right\}$ and $\left\{S_{a}\right\}$ be two asymptotically equivalent subdivision schemes having finite masks of the same support. Suppose $\left\{S_{a_{k}}\right\}$ is non-stationary and $\left\{S_{a}\right\}$ is stationary. If $\left\{S_{a}\right\}$ is $C^{m}$ and

$$
\sum_{k=0}^{\infty} 2^{m k}\left\|S_{a_{k}}-S_{a}\right\|_{\infty}<\infty
$$

then the non-stationary scheme $\left\{S_{a_{k}}\right\}$ is $C^{m}$.

\section{The subdivision scheme}

Define a space of trigonometric polynomials $\mathcal{T}$ by

$$
\mathcal{T}:=\operatorname{span}\{1, c(x), s(x), c(2 x), s(2 x)\},
$$

where $c(x)=\cos (\alpha x)$ and $s(x)=\sin (\alpha x)$ for some $\alpha$ such that $0 \leq$ $\alpha<\pi$.

Let $g \in \mathcal{T}$ and $g(x)=a_{0}+a_{1} c(x)+a_{2} s(x)+a_{3} c(2 x)+a_{4} s(2 x)$. Then the sum $a_{3}^{2}+a_{4}^{2}$ is called the amplitude of $g$.

Suppose we have a data set

$$
D=\left\{\left(x_{0}, f\left(x_{0}\right)\right),\left(x_{1}, f\left(x_{1}\right)\right),\left(x_{2}, f\left(x_{2}\right)\right),\left(x_{3}, f\left(x_{3}\right)\right)\right\} .
$$

Then there exist several functions in $\mathcal{T}$ interpolating the data set $D$. But it is known [6] that the function

$$
L(x)=\sum_{j=0}^{3} f\left(x_{j}\right) L_{j}(x)
$$

where

$$
L_{j}(x)=c\left(\frac{x-x_{j}}{2}\right) \prod_{k=0, k \neq j}^{3} \frac{s\left(\frac{x-x_{k}}{2}\right)}{s\left(\frac{x_{j}-x_{k}}{2}\right)}
$$


is a unique function in $\mathcal{T}$ which interpolates $D$ and has the minimum amplitude among other interpolants from $\mathcal{T}$. We call the function $L(x)$ a Lagrange-like interpolant of the above data.

Define $x_{j}=j, j=0,1,2,3$ and $x^{\prime}=3 / 2$, then

$$
\begin{aligned}
& L_{0}\left(x^{\prime}\right)=L_{3}\left(x^{\prime}\right)=-\frac{\sin ^{2}(\alpha / 4)}{2 \sin (\alpha / 2) \sin (\alpha)}, \\
& L_{1}\left(x^{\prime}\right)=L_{2}\left(x^{\prime}\right)=\frac{\sin ^{2}(3 \alpha / 4)}{2 \sin (\alpha / 2) \sin (\alpha)} .
\end{aligned}
$$

Clearly

$$
\frac{\sin ^{2}(3 \alpha / 4)}{\sin (\alpha / 2) \sin (\alpha)}-\frac{\sin ^{2}(\alpha / 4)}{\sin (\alpha / 2) \sin (\alpha)}=1
$$

Let $w_{0}=\sin ^{2}(\alpha / 4) /[2 \sin (\alpha / 2) \sin \alpha]$, then

$$
\frac{1}{2}+w_{0}=\frac{\sin ^{2}(3 \alpha / 4)}{2 \sin (\alpha / 2) \sin \alpha}
$$

and

$$
L\left(x^{\prime}\right)=-w_{0}\left[f\left(x_{0}\right)+f\left(x_{3}\right)\right]+\left(\frac{1}{2}+w_{0}\right)\left[f\left(x_{1}\right)+f\left(x_{2}\right)\right] .
$$

To define our non-stationary scheme, for $k \geq 0$, we denote

$$
w_{k}=\frac{\sin ^{2}\left(\frac{\alpha}{2^{k+2}}\right)}{2 \sin \left(\frac{\alpha}{2^{k}}\right) \sin \left(\frac{\alpha}{2^{k+1}}\right)}=\frac{1}{16 \cos ^{2}\left(\frac{\alpha}{2^{k+2}}\right) \cos \left(\frac{\alpha}{2^{k+1}}\right)} .
$$

Some estimates of $w_{k}$ which are useful in our scheme are given in the following lemma.

Lemma 5 For $k \geq 0$ and $0 \leq \alpha \leq \pi / 2$ :

1. $\frac{1}{8} \geq w_{k} \geq \frac{1}{16}$;

2. $\left|w_{k}-\frac{1}{16}\right| \leq C / 2^{2 k}$ for some constant $C$ independent of $k$. 
Proof: The inequality $w_{k} \geq \frac{1}{16}$ follows directly from (1). Observe that

$$
w_{k}=\frac{1}{16 \cos ^{2}\left(\frac{\alpha}{2^{k+2}}\right) \cos \left(\frac{\alpha}{2^{k+1}}\right)} \leq \frac{1}{8\left(\cos ^{2}\left(\frac{\alpha}{2^{k+1}}\right)+\cos \left(\frac{\alpha}{2^{k+1}}\right) \text { Bigr }\right)} \leq \frac{1}{8}
$$

since $\cos ^{2}(x)+\cos (x)>1$ for all $x \in[0, \pi / 4]$. This proves 1 . Also note that

$$
\begin{aligned}
w_{k}-\frac{1}{16} & =\frac{1}{16}\left(\frac{1-\cos ^{2}\left(\frac{\alpha}{2^{k+2}}\right) \cos \left(\frac{\alpha}{2^{k+1}}\right)}{\cos ^{2}\left(\frac{\alpha}{2^{k+2}}\right) \cos \left(\frac{\alpha}{2^{k+1}}\right)}\right) \\
& =\frac{\left(2+\cos \left(\frac{\alpha}{2^{k+1}}\right)\right) \sin ^{2}\left(\frac{\alpha}{2^{k+2}}\right)}{16 \cos ^{2}\left(\frac{\alpha}{2^{k+2}}\right) \cos \left(\frac{\alpha}{2^{k+1}}\right)} \\
& \leq \frac{3 \alpha^{2}}{16 \cos ^{2}(\alpha / 4) \cos (\alpha / 2)} \frac{1}{2^{2 k+4}} .
\end{aligned}
$$

The lemma follows by choosing $C=\alpha^{2} /\left(\cos ^{2}(\alpha / 4) \cos (\alpha / 2)\right)$.

We now present the basic algorithm which is a non-stationary subdivision scheme.

Algorithm: Given control points $\left\{p_{i}^{0} \in \mathbb{R} \mid i=-2,-1, \ldots, n+\right.$ $2\}$, the control points $\left\{p_{i}^{k+1} \mid i=-2,-1,0, \ldots, 2^{k+1} n+1\right\}$ at level $k+1$ are given by the following recursive relation:

$$
\begin{aligned}
p_{2 i}^{k+1}= & p_{i}^{k}, \quad-1 \leq i \leq 2^{k} n+1 ; \\
p_{2 i+1}^{k+1}= & -w_{k} p_{i-1}^{k}+\left(\frac{1}{2}+w_{k}\right) p_{i}^{k}+\left(\frac{1}{2}+w_{k}\right) p_{i+1}^{k}-w_{k} p_{i+2}^{k}, \\
& -1 \leq i \leq 2^{k} n .
\end{aligned}
$$

Remark: If we take $w_{k}=\frac{1}{16}$ for all $k$, then this scheme coincides with Dyn and Levin's four point subdivision scheme [1, 4]. The 
point $\left((2 i+1) / 2^{k+1}, p_{2 i+1}^{k+1}\right)$ lies on the Lagrange-like interpolant of the points

$$
\left\{\left(\frac{i-1}{2^{k}}, p_{i-1}^{k}\right),\left(\frac{i}{2^{k}}, p_{i}^{k}\right),\left(\frac{i+1}{2^{k}}, p_{i+1}^{k}\right),\left(\frac{i+2}{2^{k}}, p_{i+2}^{k}\right)\right\} .
$$

Note that the set of points at the $(k+1)$ th level of the algorithm contains all the points at the $k$ th level and some new points. Therefore the initial set of control points is contained at all levels of the algorithm. Therefore the limit curve interpolates the set of initial points.

Let us denote our non-stationary scheme by $\left\{S_{k}\right\}$. The mask of $\left\{S_{k}\right\}$ at the $k$ th level is $a^{(k)}=\left\{a_{-3}^{(k)}, \ldots, a_{3}^{(k)}\right\}$ where

$$
\begin{aligned}
& a_{-3}^{(k)}=a_{3}^{(k)}=-w_{k}, \quad a_{-2}^{(k)}=a_{2}^{(k)}=0, \\
& a_{0}^{(k)}=1, \quad a_{-1}^{(k)}=a_{1}^{(k)}=1 / 2+w_{k} .
\end{aligned}
$$

Note that the subdivision operator $S$ associated with the four point scheme of Dyn and Levin has the mask $a=\left\{a_{-3}, \ldots, a_{3}\right\}$ where

$$
a_{-3}=a_{3}=-\frac{1}{16}, \quad a_{-2}=a_{2}=0, \quad a_{0}=1, \quad a_{-1}=a_{1}=\frac{9}{16} .
$$

Below we establish the asymptotic equivalence of $\left\{S_{k}\right\}$ and $\{S\}$.

Theorem 6 The non-stationary scheme $\left\{S_{k}\right\}$ is asymptotically equivalent to the stationary scheme $\{S\}$. Moreover, the limit function belongs to $C^{1}(\mathbb{R})$.

Proof: We have

$$
\sum_{\beta \in \mathbb{Z}}\left|a_{2 \beta}^{(k)}-a_{2 \beta}\right|=0 \quad \text { and } \quad \sum_{\beta \in \mathbb{Z}}\left|a_{1+2 \beta}^{(k)}-a_{1+2 \beta}\right|=4\left|w_{k}-1 / 16\right| .
$$


By Lemma 5, Part 2, we get $\sum_{\beta \in \mathbb{Z}}\left|a_{1+2 \beta}^{(k)}-a_{1+2 \beta}\right| \leq \frac{4 C}{2^{2 k}}$ and hence

$$
\left\|S_{k}-S\right\| \leq \frac{4 C}{2^{2 k}}
$$

Hence $\sum_{k=0}^{\infty}\left\|S_{k}-S\right\|_{\infty}<\infty$ and the schemes $\left\{S_{k}\right\}$ and $\{S\}$ are asymptotically equivalent. It is clear that

$$
\sum_{k=0}^{\infty} 2^{k}\left\|S_{k}-S\right\|_{\infty}<\infty .
$$

As the scheme associated with $S$ is $C^{1}$, then by Theorem 4 the scheme $\left\{S_{k}\right\}$ is also $C^{1}$. This proves the theorem.

\section{Basic limit function}

The basic limit function of the scheme $\left\{S_{k}\right\}$ is the limit function of the scheme for the data

$$
p_{i}^{0}= \begin{cases}1, & i=0, \\ 0, & i \neq 0 .\end{cases}
$$

By Theorem 6, the basic limit function denoted below by $F$ belongs to $C^{1}(\mathbb{R})$. In this section we derive some basic properties of the basic limit function $F$. Let

$$
D_{n}:=\left\{j / 2^{n} \mid j \in \mathbb{Z}\right\} .
$$

It is easy to check that restriction of $F$ to $D_{n}$ satisfies $F\left(\frac{j}{2^{n}}\right)=p_{j}^{n}$ for all $j$.

Theorem $7 F$ is symmetric about the $Y$-axis. 
Proof: We prove this by induction on $n$. First of all $F\left(\frac{j}{2^{n}}\right)=$ $F\left(-\frac{j}{2^{n}}\right)$ for $n=0$ since $F(j)=F(-j)=0, j \in \mathbb{Z}$, by the interpolation property. Assume that $F\left(\frac{j}{2^{k}}\right)=F\left(-\frac{j}{2^{k}}\right), j \in \mathbb{Z}$, $k=1,2, \ldots, n$. Therefore $F\left(\frac{2 j}{2^{n+1}}\right)=F\left(-\frac{2 j}{2^{n+1}}\right)$ for all $j$. Moreover,

$$
\begin{aligned}
F\left(\frac{2 j+1}{2^{n+1}}\right) & \\
=- & w_{n} F\left(\frac{j-1}{2^{n}}\right)+\left(1 / 2+w_{n}\right) F\left(\frac{j}{2^{n}}\right) \\
& \quad+\left(1 / 2+w_{n}\right) F\left(\frac{j+1}{2^{n}}\right)-w_{n} F\left(\frac{j+2}{2^{n}}\right) \\
=- & w_{n} F\left(\frac{-j+1}{2^{n}}\right)+\left(1 / 2+w_{n}\right)\left[F\left(\frac{-j}{2^{n}}\right)+F\left(\frac{-j-1}{2^{n}}\right)\right] \\
& \quad-w_{n} F\left(\frac{-j-2}{2^{n}}\right) \\
= & F\left(-\frac{2 j+1}{2^{n+1}}\right) .
\end{aligned}
$$

Hence $F\left(\frac{j}{2^{n}}\right)=F\left(-\frac{j}{2^{n}}\right)$ for all $j$ and $n \in \mathbb{Z}$. From the continuity of $F$ we have $F(x)=F(-x)$ for all $x \in \mathbb{R}$ which completes the proof of the theorem.

The translation $F(\cdot-k), k \in \mathbb{R}$ of $F$ is the limit function of the scheme for the initial data

$$
p_{i}^{0}= \begin{cases}1, & i=k \\ 0, & i \neq k\end{cases}
$$

Next we show that $F$ is a compactly supported function with support in $[-3,3]$, see Figures 1 and 2.

Theorem 8 The basic limit function $F$ vanishes outside $[-3,3]$. 


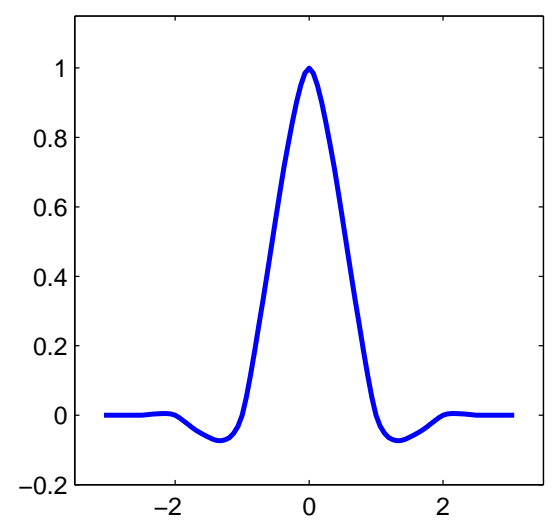

(a)

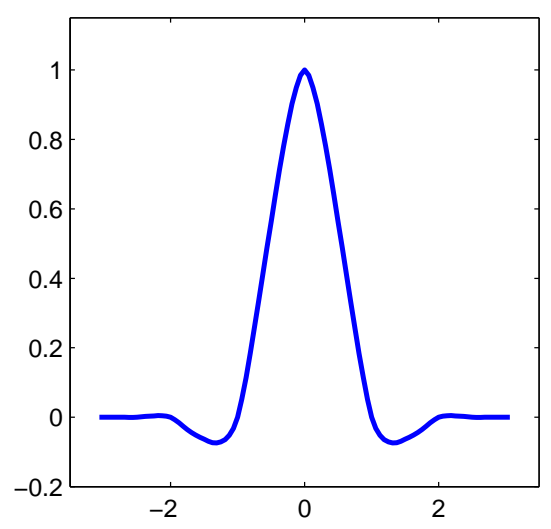

(b)

Figure 1: Basic limit functions: (a) for $\alpha=0.25$ and (b) for $\alpha=$ 0.5 respectively.

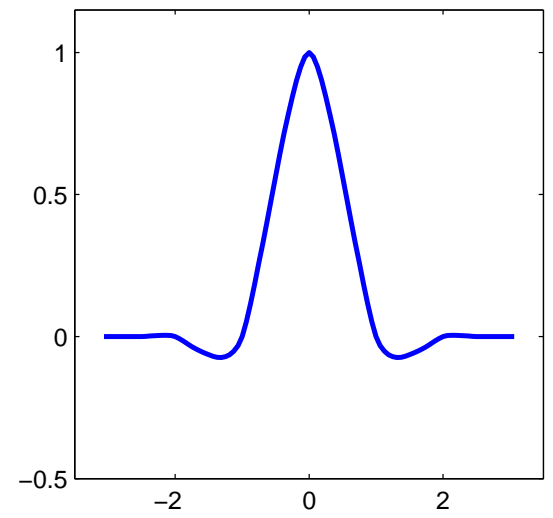

(a)

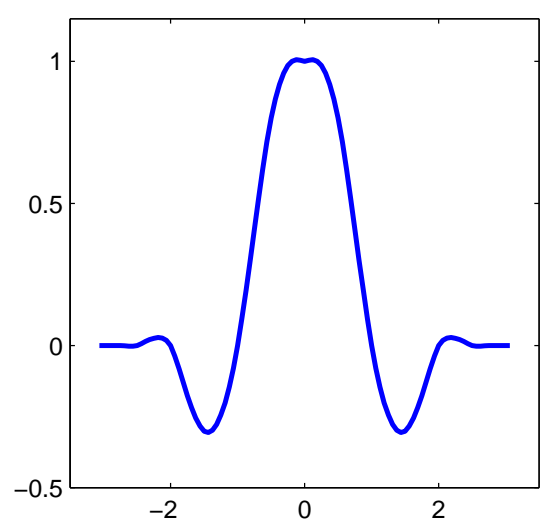

(b)

Figure 2: Basic limit functions: (a) for $\alpha=1.0$ and (b) for $\alpha=2.5$ respectively. 
Proof: Take $t_{0}=0$ and define $t_{n}$ recursively by $t_{n+1}=t_{n}+\frac{3}{2^{n+1}}$. Observe that $t_{n} \in D_{n}$ but $t_{n} \notin D_{n-1}$. We claim that the restriction of $F$ to $D_{n}$ vanishes outside $\left[-t_{n}, t_{n}\right]$. Since $F$ is symmetric it is enough to prove that $F$ vanishes outside $\left[0, t_{n}\right]$. We prove this by induction on $n$. Observe that

$$
\begin{aligned}
F & \left(t_{n+1}+\frac{2 k}{2^{n+1}}\right) \\
= & F\left(t_{n}+\frac{2 k+3}{2^{n+1}}\right) \\
= & -w_{n}\left[F\left(t_{n}+\frac{k}{2^{n}}\right)+F\left(t_{n}+\frac{k+3}{2^{n}}\right)\right] \\
& +\left(1 / 2+w_{n}\right)\left[F\left(t_{n}+\frac{k+1}{2^{n}}\right)+F\left(t_{n}+\frac{k+2}{2^{n}}\right)\right]
\end{aligned}
$$

and

$$
F\left(t_{n+1}+\frac{2 k+1}{2^{n+1}}\right)=F\left(t_{n}+\frac{k+2}{2^{n}}\right) .
$$

From the above mentioned equation it is easy to check that $F\left(t_{1}\right)=$ $-w_{0} F(0) \neq 0$ and for $k>0$

$$
F\left(t_{1}+\frac{2 k-1}{2}\right)=0 \text { and } F\left(t_{1}+\frac{2 k}{2}\right)=0 .
$$

This proves our claim for $n=1$.

Assume $F(x)=0$ whenever $x \in D_{n}$ and $x>t_{n}$. Then by (2) and (3) we get $F\left(t_{n+1}\right)=-w_{n} F\left(t_{n}\right) \neq 0$ and for $k>0$ we have $F\left(t_{n+1}+\frac{2 k}{2^{n+1}}\right)=0$ and $F\left(t_{n+1}+\frac{2 k-1}{2^{n+1}}\right)=0$. Since $\left[-t_{n}, t_{n}\right]$ is contained in $[-3,3]$ for all $n$ and $\lim _{n \rightarrow \infty}\left[-t_{n}, t_{n}\right]=[-3,3]$ we have the required result. 
Remark: Since $F$ is compactly supported and continuous on $\mathbb{R}$, it follows that $F(\cdot-k)$ are also compactly supported continuous functions. If $y(t)$ is the limit function interpolating the initial sequence $\{y(n)\}$, then

$$
y(t)=\sum_{j \in \mathbb{Z}} y(j) F(t-j)=\sum_{j=k-2}^{k+3} y(j) F(t-j)
$$

where $k$ is the greatest integer such that $k \leq t$.

\section{$5 \quad$ Reconstruction of functions}

In this section we show that certain functions can be reconstructed by our scheme. It is easy to check that if $p_{i}^{k}=1$ for all $i$ at the $k$ th level then $p_{j}^{k+1}=1$ for all $j$ at the $(k+1)$ th level. This shows that the function $f(x)=1$ is reproduced by our scheme. Another simple consequence is

$$
\sum_{k \in \mathbb{Z}} F(t-k)=1, \quad t \in \mathbb{R} .
$$

Therefore the translations of the basic limit function $F$ form a partition of unity.

The functions $\cos (\alpha x)$ and $\sin (\alpha x)$ can also be reconstructed by our scheme which follows from the next lemma.

Lemma 9 Let $k \geq 0$ and $n>0$ be fixed integers. Let $p_{j}^{k}=$ $\cos \left(j \frac{\alpha}{2^{k}}\right),-2 \leq j \leq 2^{k} n+2$. Then we have for $-1 \leq i \leq 2^{k} n$,

$$
p_{2 i}^{k+1}=\cos \left(\frac{2 i \alpha}{2^{k+1}}\right) \quad \text { and } \quad p_{2 i+1}^{k+1}=\cos \left((2 i+1) \frac{\alpha}{2^{k+1}}\right) .
$$


Similarly, if $p_{j}^{k}=\sin \left(j \frac{\alpha}{2^{k}}\right)$, then for $-1 \leq i \leq 2^{k} n$,

$$
p_{2 i}^{k+1}=\sin \left(\frac{2 i \alpha}{2^{k+1}}\right) \quad \text { and } \quad p_{2 i+1}^{k+1}=\sin \left((2 i+1) \frac{\alpha}{2^{k+1}}\right) \text {. }
$$

Proof: We present the proof of the first part and the proof of the second part follows by a similar argument. Note that

$$
p_{2 i}^{k+1}=p_{i}^{k}=\cos \left(i \frac{\alpha}{2^{k}}\right)=\cos \left(2 i \frac{\alpha}{2^{k+1}}\right)
$$

and

$$
p_{2 i+1}^{k+1}=-w_{k}\left(p_{i-1}^{k}+p_{i+2}^{k}\right)+\left(1 / 2+w_{k}\right)\left(p_{i}^{k}+p_{i+1}^{k}\right) .
$$

Since

$$
\begin{aligned}
\frac{1}{2}+w_{k} & =\frac{\sin ^{2}\left(3 \frac{\alpha}{2^{k+2}}\right)}{2 \sin \left(\frac{\alpha}{2^{k+1}}\right) \sin \left(\frac{\alpha}{2^{k}}\right)}, \\
p_{i}^{k}+p_{i+1}^{k} & =2 \cos \left((2 i+1) \frac{\alpha}{2^{k+1}}\right) \cos \left(\frac{\alpha}{2^{k+1}}\right)
\end{aligned}
$$

and

$$
p_{i-1}^{k}+p_{i+2}^{k}=2 \cos \left((2 i+1) \frac{\alpha}{2^{k+1}}\right) \cos \left(3 \frac{\alpha}{2^{k+1}}\right),
$$

we get

$$
\begin{aligned}
p_{2 i+1}^{k+1}= & {\left[\frac{-2 \sin ^{2}\left(\frac{\alpha}{2^{k+2}}\right)}{2 \sin \left(\frac{\alpha}{2^{k+1}}\right) \sin \left(\frac{\alpha}{2^{k}}\right)} \cos \left(3 \frac{\alpha}{2^{k+1}}\right)\right.} \\
& \left.+\frac{2 \sin ^{2}\left(3 \frac{\alpha}{2^{k+2}}\right)}{2 \sin \left(\frac{\alpha}{2^{k+1}}\right) \sin \left(\frac{\alpha}{2^{k}}\right)} \cos \left(\frac{\alpha}{2^{k+1}}\right)\right] \cos \left(\frac{2 i+1}{2} \frac{\alpha}{2^{k}}\right) \\
= & \frac{2 \sin \left(\frac{\alpha}{2^{k+1}}\right) \sin \left(\frac{\alpha}{2^{k}}\right)}{2 \sin \left(\frac{\alpha}{2^{k+1}}\right) \sin \left(\frac{\alpha}{2^{k}}\right)} \cos \left(\frac{2 i+1}{2} \frac{\alpha}{2^{k}}\right) \\
= & \cos \left((2 i+1) \frac{\alpha}{2^{k+1}}\right) .
\end{aligned}
$$




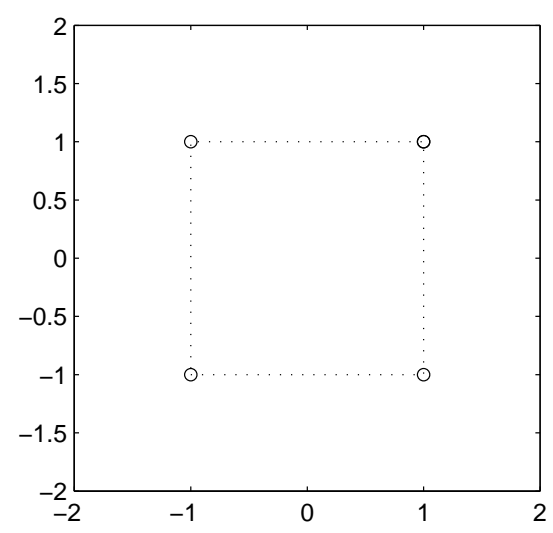

(a)

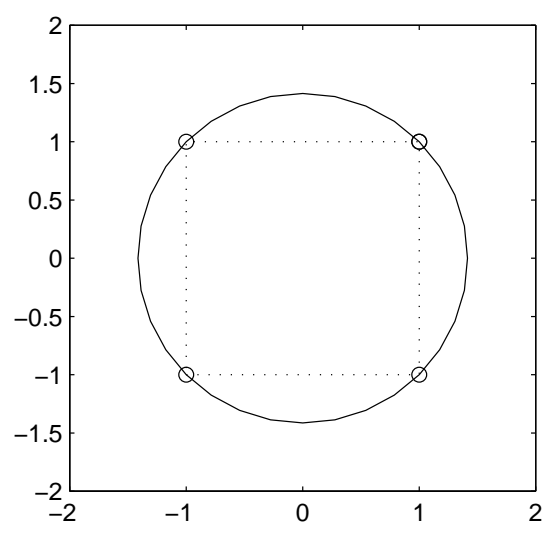

(b)

Figure 3: (a): Points on the unit square, (b): The limit curve after the third iteration.

This proves the lemma.

Corollary 10 If the initial data lie on a graph of a function $f \in \mathcal{T}$ and the values of $f$ are given on a set of equidistant points then the limit function of the non-stationary subdivision scheme exactly reproduces the original function $f$. In particular if we choose a set of equidistant points

$$
p_{i}^{0}=\left(\cos \left(k \frac{2 \pi}{n}\right), \sin \left(k \frac{2 \pi}{n}\right)\right), \quad k=0,1, \ldots, n
$$

on a circle, and $\alpha=2 \pi / n$, then the limit curve is the original unit circle (see Figure 3). 


\section{Order of approximation}

Finally, we present a result on the order of approximation of our interpolation scheme. To state the basic approximation theorem, we assume that for a fixed $n \in \mathbb{N}$ let $h=\frac{1}{n}$ and $I=[-2 h, 1+h]$. Suppose that $g$ is a $C^{2}(I)$ function defined on $I$ and

$$
p_{i}^{0}=g(i h), \quad-2 \leq i \leq n+2 .
$$

The basic approximation theorem states that the limit function obtained by the above non-stationary scheme with the data $g(i h)$, $-2 \leq i \leq n+2$ approximates $g(t)$ with an error of $O\left(h^{2}\right)$. We now state the theorem precisely.

Theorem 11 Let $p_{i}^{0}=g(i h),-2 \leq i \leq n+2, h=1 / n$. Let $f$ be the limit function of the non-stationary interpolatory scheme. If $g \in C^{2}(I)$ then there exists a constant $C$ such that

$$
\|f-g\|_{\infty,[0,1]}:=\max _{0 \leq x \leq 1}|f(x)-g(x)| \leq C \frac{h^{2}}{\cos (5 \alpha h / 2)} .
$$

Proof: Define the compactly supported functions $B_{i}, i \in \mathbb{Z}$ by

$$
B_{i}(x):=F\left(\frac{x}{h}-i\right), \quad i \in \mathbb{Z},
$$

where $F$ is the basic limit function of the nonstationary scheme. By (5)

$$
\sum_{i \in \mathbb{Z}} B_{i}(x)=1, \quad \forall x \in I .
$$

Denote the interval $[i h, j h]$ by $I_{i, j}$. Then for $x \in I_{k, k+1}$, by (4), we have

$$
f(x)=\sum_{i=k-2}^{k+3} p_{i}^{0} B_{i}(x)=\sum_{i=k-2}^{k+3} g(i h) B_{i}(x) .
$$


Define

$$
Q(x)=\frac{\sin (\alpha((k+3) h-x))}{\sin (5 \alpha h)} p_{k-2}^{0}+\frac{\sin (\alpha(x-(k-2) h))}{\sin (5 \alpha h)} p_{k+3}^{0} .
$$

It is easy to check that $Q \in \mathcal{T}$ and hence by Corollary 10 and (4), for $x \in I_{k-2, k+3}$ we get

$$
Q(x)=\sum_{i=k-2}^{k+3} Q(i h) F\left(\frac{x}{h}-i\right) .
$$

Koch [5] showed that if $g \in C^{2}(I)$ then there exists a constant $C_{1}$ depending only upon $g$ such that

$$
\|Q-g\|_{\infty, I_{k-2, k+3}} \leq C_{1} \frac{h^{2}}{\cos (5 \alpha / 2)} .
$$

Moreover, we have

$$
\begin{aligned}
\|f-Q\|_{\infty, I_{k, k+1}} & =\left\|\sum_{j=k-2}^{k+3} g(j h) B_{j}(x)-\sum_{j=k-2}^{k+3} Q(j h) B_{j}(x)\right\|_{\infty, I_{k, k+1}} \\
& \leq 5 \max _{k-2 \leq j \leq k+3}\left\|B_{j}(x)\right\|_{\infty, I_{k-2, k+3}}\|Q-g\|_{\infty, I_{k-2, k+3}} .
\end{aligned}
$$

Since the basic limit functions $B_{j}(x), j \in \mathbb{Z}$ are bounded functions we have

$$
\begin{aligned}
\|f-g\|_{\infty, I_{k, k+1}} \leq & \|f-Q\|_{\infty, I_{k, k+1}}+\|Q-g\|_{\infty, I_{k, k+1}} \\
& \leq \max \left\{5\left\|B_{j}(x)\right\|_{\infty, I_{k, k+1}}+1\right\} C_{1} \frac{h^{2}}{\cos (5 \alpha h / 2)} .
\end{aligned}
$$

This completes the proof of the theorem. 


\section{Discussion}

Some recently developed schemes generate functions spanned by sines and cosines, and reproduce circles. By using a mixed set of polynomials and trigonometric basis elements, Zhang [10] introduced C-Bezier and C-spline curves. He also presented a nonstationary subdivision scheme for $\mathrm{C}$-spline curves which is capable of reproducing circles and generating arcs of ellipses. These types of problems have been solved mostly using Non Uniform Rational B-splines. However, our scheme and the scheme for the C-splines approach this problem in two different ways. The scheme introduced in [10] for C-splines is $C^{2}$; however, unlike our $C^{1}$ scheme it is not interpolatory. Besides, our scheme in view of its interpolatory and local nature has distinct advantages. Moreover, formulas entering into our scheme are quite simple. The non-stationary $C^{2}$ scheme introduced in [7] unifies the known subdivision schemes for cubic B-splines and the scheme introduced in [10]. However, it is again not interpolatory.

Our subdivision scheme can be naturally used for the design of interpolatory surfaces using a tensor product approach. Further generalisation of this scheme for generating surfaces from arbitrary meshes is a different issue and is beyond the scope of this paper.

Acknowledgement: We thank the referee for comments which helped us to improve the content and the presentation of the paper. 


\section{References}

[1] S. Dubuc. "Interpolation through an iterative Scheme". Journal of Mathematical Analysis and Applications, 114:185-204, 1986. E218, E222

[2] N. Dyn. "Subdivision schemes in computer aided geometric design". In W.A. Light, editor, Advances in Numerical Analysis II, Wavelets, Subdivision Algorithms and Radial Functions, pages 36-104. Oxford University Press, 1992. E217

[3] N. Dyn and D. Levin. "Analysis of asymptotically equivalent binary subdivision schemes". Journal of Mathematical Analysis and Applications, 193:594-621, 1995. E217, E219

[4] N. Dyn, D. Levin, and J.A. Gregory. " 4-point interpolatory subdivision scheme for curve design". Computer Aided Geometric Design, 4:257-268, 1987. E218, E222

[5] P.E. Koch, T. Lyche, M. Neamtu, and L. Schumaker. "Control curves and knot insertion for trigonometric splines". Advances in Computational Mathematics, 3:405-424, 1995. $\mathrm{E} 232$

[6] T. Lyche. "A Newton form for trigonometric Hermite interpolation". BIT , 19:229-235, 1979. E220

[7] G. Morin, J. Warren, and H. Weimer. "A subdivision scheme for surfaces of revolution". Computer Aided Geometric Design, 18:483-502, 2001. E233

[8] E.J. Stollnitz, T.D. DeRose, and D.H. Salesin. Wavelet For Computer Graphics, Theory and Applications. Morgan 
Kaufmann Publishers,Inc., San Francisco, California, 1995. E217

[9] H. Weimer and J. Warren. "Non-stationary subdivision for nonhomogeneous operator differential equations". In P. Sabloniere, L.L. Schumaker, and P.J. Laurent, editors, Curve and Surface Fitting: Saint-Malo 99. Vanderbilt University Press, 2000. E217

[10] J. Zhang. "C-curves : An extension of cubic curves". Computer Aided Geometric Design, 13:199-217, 1996. E233 